\title{
Cancer-Associated Retinopathy due to Clear Cell Renal Carcinoma
}

\author{
Sushant Wagley ${ }^{a}$ Tu M. Tran ${ }^{a}$ Paul W. Mallory ${ }^{a}$ Michael S. Lee ${ }^{a}$ \\ Karen R. Armbrust ${ }^{b}$ Bruce Trautman ${ }^{c}$ Sandra R. Montezuma ${ }^{a}$ \\ ${ }^{a}$ Department of Ophthalmology and Visual Neurosciences, University of Minnesota, Minneapolis, MN, USA; \\ bVA Health Care System, Minneapolis, MN, USA; ' $A$ Anatomic Pathology, Fairview Southdale Hospital, Edina, MN, USA
}

\section{Established Facts}

- Cancer-associated retinopathy manifests with bilateral, progressive cone, and rod dysfunction.

- Symptoms of cancer-associated retinopathy often precede diagnosis of malignancy.

\section{Novel Insights}

- Clear cell type renal cell carcinoma can be a cause of cancer-associated retinopathy.

- Treatment of underlying malignancy and first-line immunosuppression may not lead to visual recovery.

\section{Keywords}

Autoimmune retinopathy - Cancer-associated retinopathy Goldmann visual field · Paraneoplastic syndrome · Renal carcinoma

\begin{abstract}
An 84-year-old female presented with bilateral scotomas and progressive nyctalopia over 1 year. Best-corrected visual acuity was 20/50 in both eyes with reduced color vision. Goldmann visual field showed bilateral cecocentral scotomas and generalized constriction of the visual fields. This led to an electroretinogram showing an electronegative pattern consistent with autoimmune retinopathies. Infectious workup was negative. Anti-retinal antibodies were positive, leading to a presumed diagnosis of cancer-associated retinopathy (CAR). Imaging showed a previously unknown left renal
\end{abstract}

lower pole mass, and she underwent a radical nephrectomy. Biopsy showed nuclear grade-3 clear cell renal carcinoma staged T1. The patient was treated with oral prednisone with no ocular improvement. We report on a rare case of clear cell renal carcinoma causing CAR. CAR is an important paraneoplastic syndrome to diagnose since the majority of ocular cases precede other manifestations of malignancy. Therefore, a timely diagnosis of CAR can be lifesaving or at least life-extending.

(c) 2020 S. Karger AG, Basel

\section{Introduction}

Cancer-associated retinopathy (CAR) is an important paraneoplastic syndrome to diagnose as it is not only rapidly progressive with poor visual recovery, but also visual
Sandra R. Montezuma

Department of Ophthalmology and Visual Neurosciences University of Minnesota, MMC 493, 420 Delaware St. SE Minneapolis, MN 55455 (USA)

smontezu@umn.edu 
symptoms precede other manifestations of an underlying malignancy [1]. The pathogenesis is thought to involve molecular mimicry generating antibodies against tumor cells, such as anti-recoverin, which then cross the bloodretina barrier to bind onto respective epitopes on photoreceptors, leading to caspase-dependent apoptosis [2]. Small-cell lung cancer is the most common malignancy associated with CAR. In this paper, we present a rare case of CAR secondary to renal cell carcinoma (RCC).

\section{Case Presentation}

An 84-year-old female presented for evaluation of bilateral central scotomas and progressive nyctalopia lasting 1 year. Ocular history included bilateral pseudophakia, ocular hypertension, and laser retinopexy for operculated retinal hole in the right eye (RE) and retinal tear in the left eye (LE). Medical history was significant for persistent atrial fibrillation, hypertension, dyslipidemia, chronic kidney disease stage IV, obstructive sleep apnea, and osteoporotic hip fracture. There was no family history of degenerative retinal disease.

On examination, best-corrected visual acuity was $20 / 50$ in both eyes. Ishihara color plates were 4/11 in the RE and 2/11 in the LE. Other findings included bilateral vitreous cells, subtle macular pigmentary changes, and focal peripheral chorioretinal scarring secondary to prior laser retinopexy (not shown). Macula fundus autofluorescence (FAF) demonstrated patchy hyperautofluorescence, mostly inferior to the macula with retinal pigmentary changes but otherwise unremarkable (Fig. 1a). Macular optical coherence tomography (OCT) showed disruption of the outer nuclear layer and ellipsoid zone with relative sparing of the fovea, and thinned choroid in both eyes (Fig. 1b). Goldman visual field (GVF) revealed generalized constriction and bilateral cecocentral scotomas (Fig. 1c). Full-field electroretinography (ffERG) showed an electronegative waveform in the LE with the dark-adapted 10.0 response (Fig. 2).

Syphilis and tuberculosis testing was negative. Serum anti-retinal antibody testing revealed positive anti-retinal antibodies against 23- (recoverin), 34-, 36-(GAPDH), 39-, 40-(aldolase C), 56-, 66-, and 68-kilodalton (KDa) proteins by Western blot. No breast or lung neoplasms were evident on examination and imaging; however, a chest, abdomen, pelvis computed tomography with contrast revealed a $4-\mathrm{cm}$ left renal lower pole heterogeneous mass without renal sinus invasion (Fig. 3). The patient was referred to urology and subsequently underwent laparoscopic radical left nephrectomy. Pathology showed nuclear grade- 3 clear cell RCC with free margins, and staging was pT1a (Fig. 4, 8th Edition, AJCC Staging Manual).

After surgery, an oral prednisone course starting at $40 \mathrm{mg}$ with taper over 8 weeks did not provide subjective or objective ocular improvement. Repeat ffERG showed progressive reduction in amplitudes, and GVF showed scotoma enlargement. Periocular triamcinolone injection was given, but the outcome of local corticosteroid treatment was unknown since the patient was lost to follow-up.

\section{Discussion and Conclusion}

We present this rare case of presumed CAR from RCC. The anti-retinal antibodies $23-\mathrm{kDa}$ (recoverin), $36-\mathrm{kDa}$ (GAPDH), $40-\mathrm{kDa}$ (aldolase C) in the context of histopathologically confirmed diagnosis and absence of evidence of any other malignancies provide strong evidence for CAR from RCC. To our knowledge, this is the first case of clear cell type and one of only a few reported cases of renal neoplasm causing paraneoplastic retinopathy [3-5]. This case highlights how identifying an autoimmune retinopathy (AIR) led to the diagnosis of an asymptomatic malignancy.

CAR pathogenesis is not completely understood, but presumably antibodies produced against tumor antigens cross the blood-retina barrier and cause retinal dysfunction. Small-cell lung, breast, and gynecological malignancies are the most common cancers associated with CAR, classically with positive anti-recoverin antibodies $[6,7]$. Although anti-retinal antibodies - and specifically antirecoverin - are confirmatory for the diagnosis of CAR, these antibodies are neither sensitive nor specific biomarkers. Anti-recoverin is present in only $5 \%$ of the CAR patients, and patients without ocular disease can have anti-retinal antibodies [6].

This patient presented with some signs and symptoms of classic AIR, including bilateral progressive vision loss, nyctalopia, decreased color vision, GVF constriction, and ERG abnormalities. However, the patient had a cecocentral scotoma instead of a classic ring scotoma. The FAF did not show a parafoveal ring of alternative autofluorescence, but patchy areas of hyperautofluorescence. The ERG abnormality was subtle and not symmetric where the LE's electronegative waveform suggested a post-phototransduction defect that can be seen with the AIRs and CAR. Although this patient did not have classic systemic findings associated with paraneoplastic syndromes, such as anemia, fever, cachexia, or hypercalcemia, any patient with AIR should undergo a malignancy workup [1]. In particular, our patient's age raised our level of suspicion for CAR and prompted the imaging that led to uncovering of the RCC.

Current therapy for CAR involves first treating the underlying malignancy, followed by an escalation of immunosuppression starting from corticosteroids (systemic, ocular) $[8,9]$, plasmapheresis, intravenous immunoglobulin [4], immunomodulatory medications [10], and monoclonal antibodies if refractory $[11,12]$. Visual prognosis in CAR is highly variable, from recovery within months to stabilization to progressive decline $[4,5]$. How-
Wagley/Tran/Mallory/Lee/Armbrust/ Trautman/Montezuma 

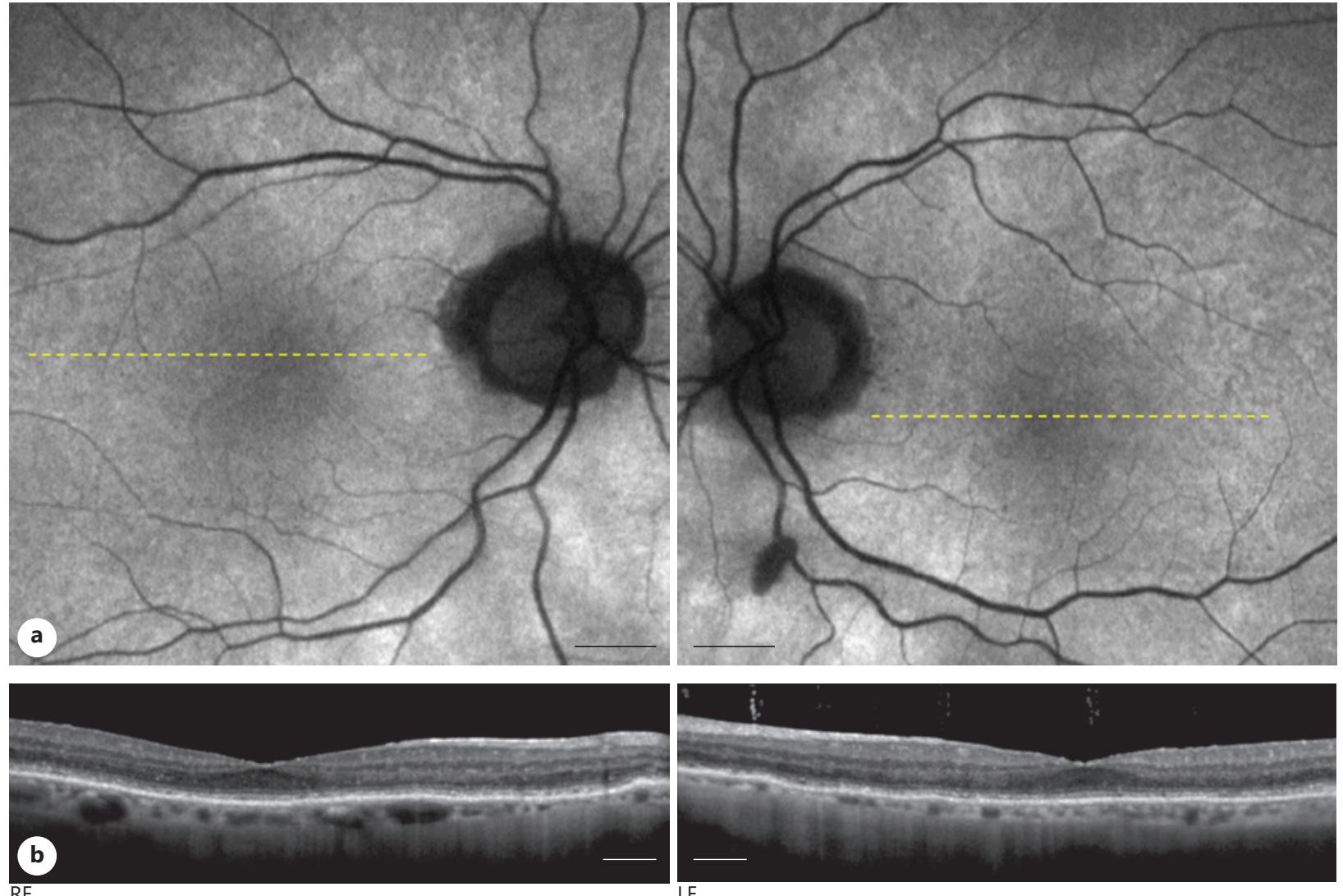

LE

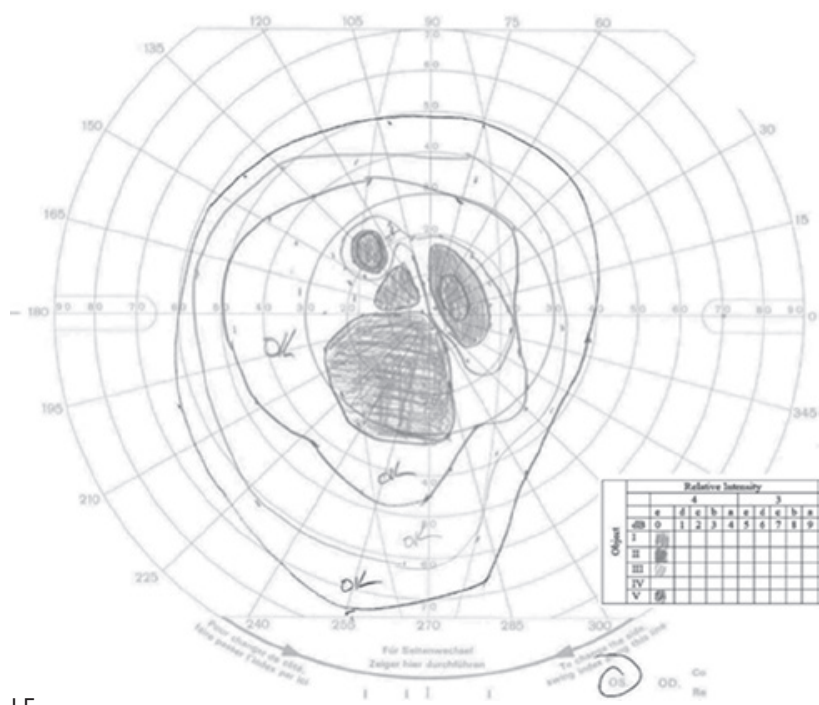

LE

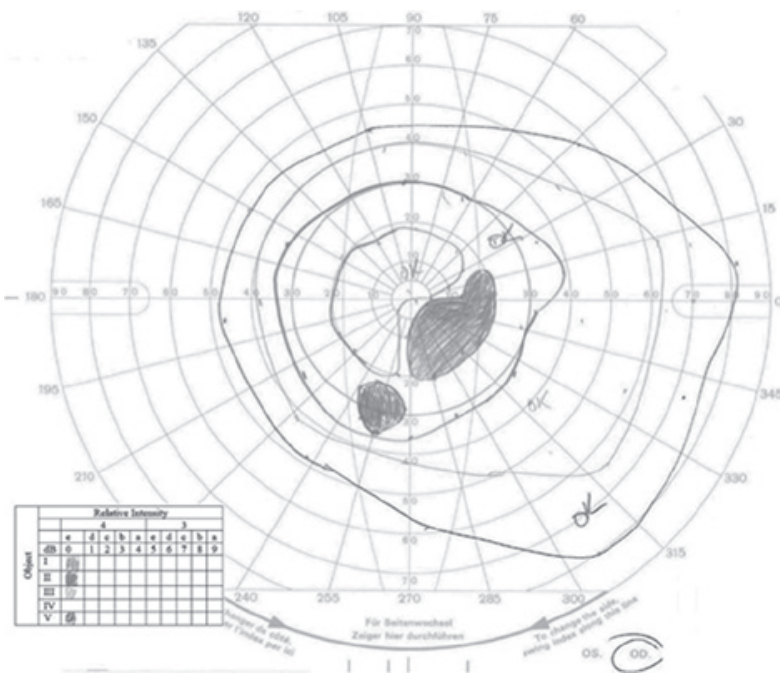

RE

Fig. 1. Imaging at initial presentation. FAF did not show ringed areas of hypo-/hyperautofluorescence in either eye; the blocked autofluorescence inferior to the optic disc in the LE correlated with a Weiss ring. Black scale bar $=500 \mu \mathrm{m}$; dotted yellow line signifies approximate location of the OCT B-scan (a). Macular OCT with increased reflectance and disruption of the outer nuclear layer and ellipsoid zone that is less pronounced at the fovea (more foveal involvement in the left than RE), and choroidal thinning. Scale bar $=200 \mu \mathrm{m}(\mathbf{b})$. GVF showing generalized constriction and cecocentral scotomas $(\mathbf{c})$. 


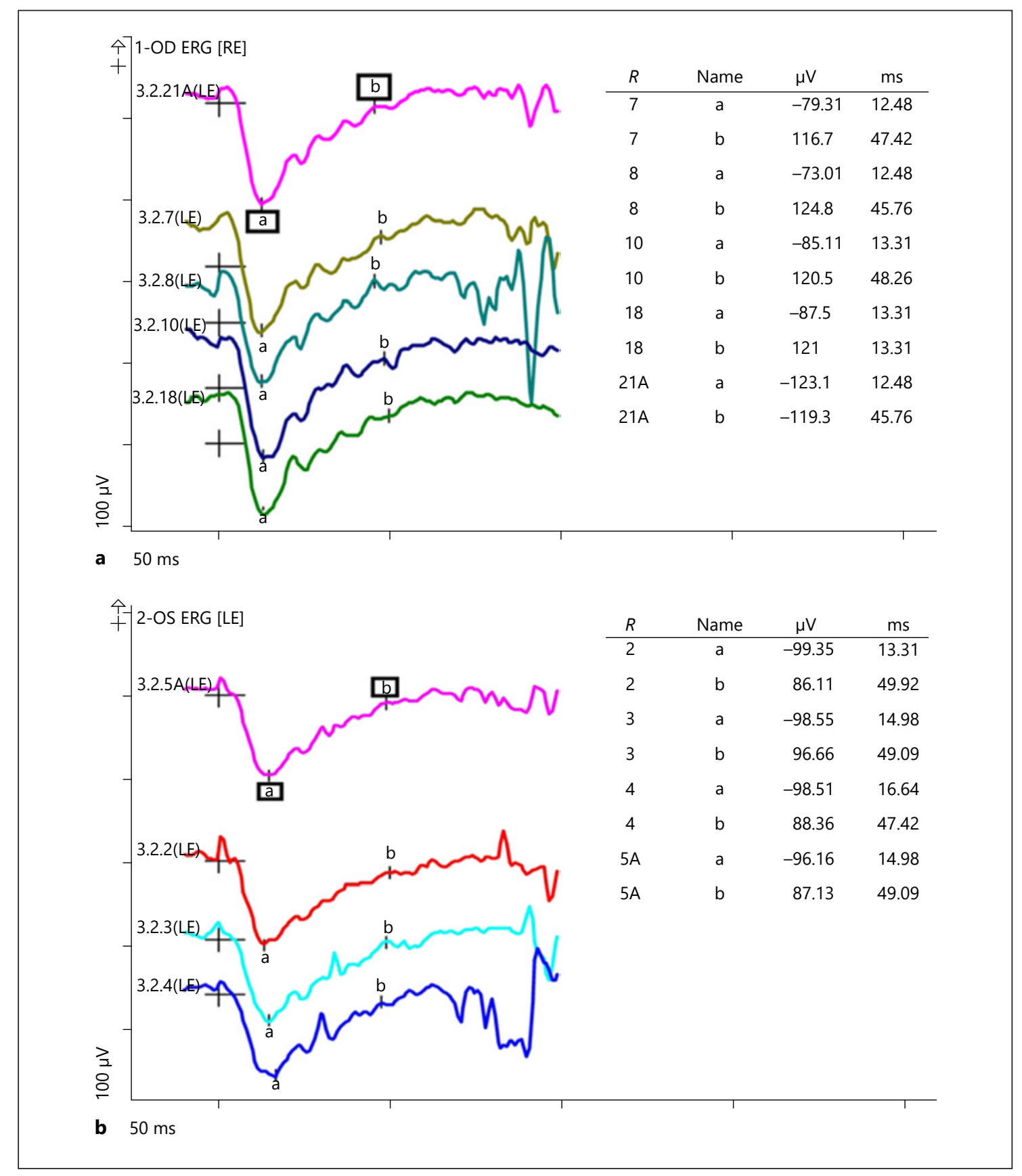

Fig. 2. ffERG, dark-adapted 10.0 with RE on top (a) and LE on the bottom (b). B-wave amplitude was lower than the a-wave in the LE. The a-waves were near normal values for our testing setup.

ever, even in cases such as this one, when visual function does not improve with treatment, merely making the diagnosis of CAR can have a substantial positive impact on systemic health. CAR may be rare, but it is an important condition for ophthalmologists to recognize since it may lead to early diagnosis of life-threatening malignancies.

\section{Statement of Ethics}

This case report adhered to the ethical standards laid out by the Declaration of Helsinki. No identifying patient information, images or likeness has been presented, and signed consent from patient and next of kin was not sought. However, the patient's next of kin provided verbal consent to publication of this case report. 


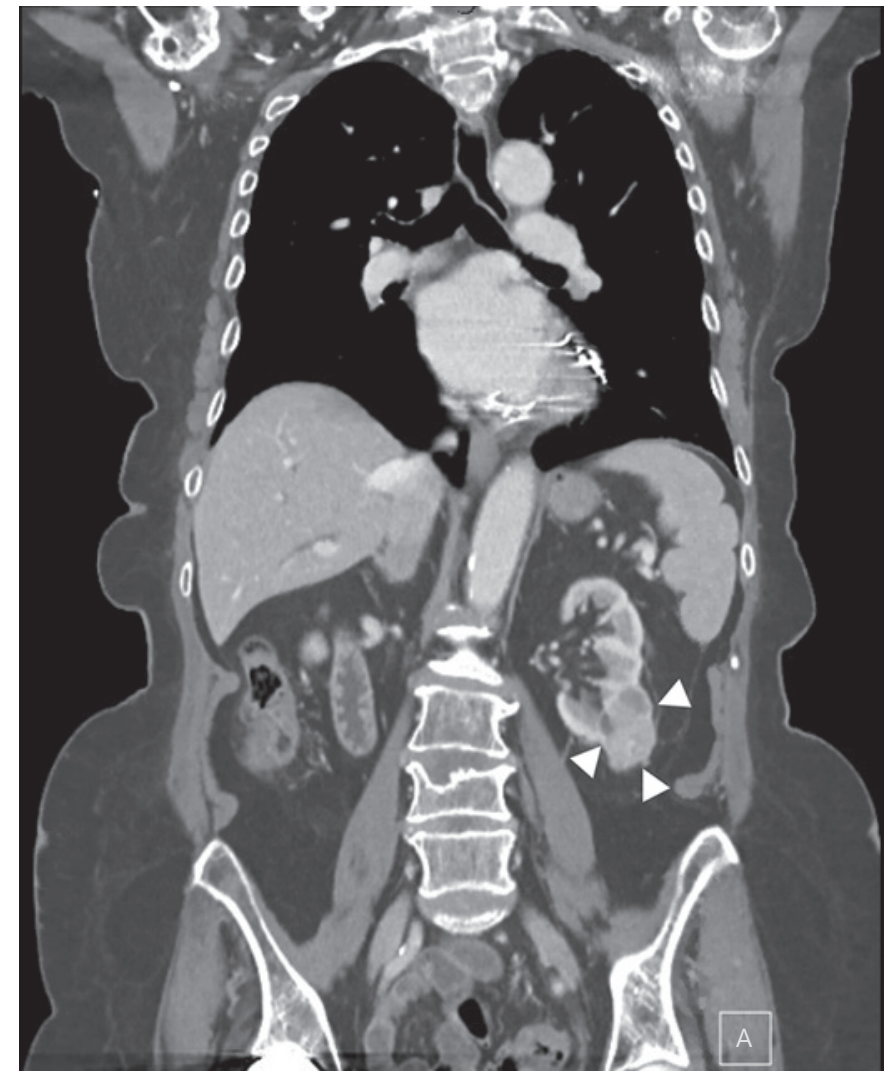

Fig. 3. Chest, abdomen, pelvis computed tomography with contrast showing a left renal mass (arrowheads).

\section{Conflict of Interest Statement}

The authors have no conflicts of interest to declare.

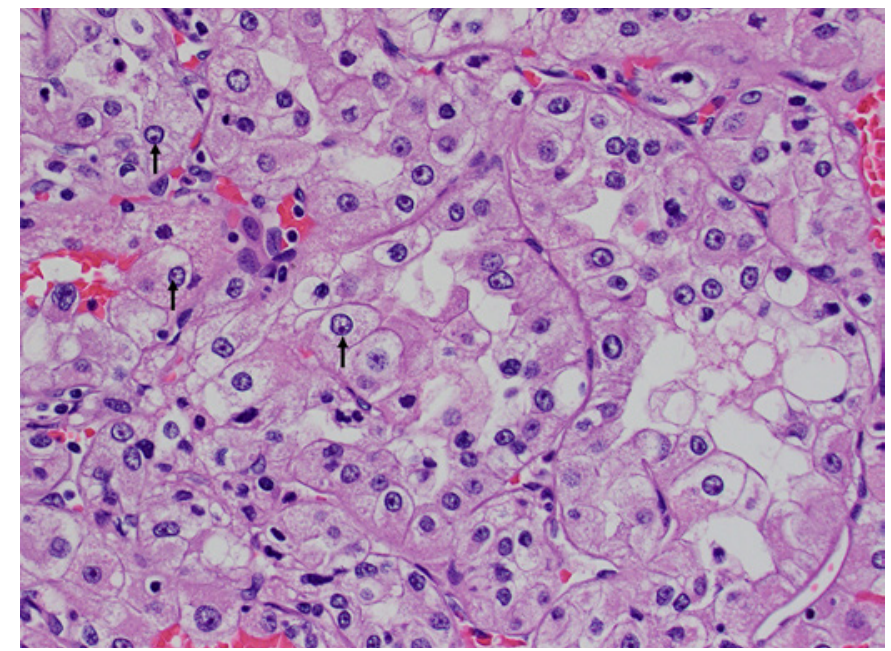

Fig. 4. Microscopy of the left renal mass showing abundant clear cytoplasm, large and irregular nuclei with open chromatin, and prominent nucleoli (arrows) consistent with Fuhrman nuclear grade 3 RCC, clear cell type $(\mathrm{H} \& \mathrm{E} . \times 40)$.

\section{Funding Sources}

No funding sources were used or involved in any way in this case report.

\section{Author Contributions}

S.W., T.M.T., and P.W.M. wrote the manuscript. S.W. and T.M.T. performed the detailed review of the patient's clinical course and the literature review. P.W.M, M.S.L., K.R.A., B.T., and S.M. were directly involved in the patient care and provided significant inputs in the composition of the manuscript.

\section{References}

1 Hoogewoud F, Butori P, Blanche P, Brézin AP. Cancer-associated retinopathy preceding the diagnosis of cancer. BMC Ophthalmol. 2018 Nov; 18(1):285.

2 Kordyś M, Przeździecka-Dołyk J, TurnoKręcicka A, Misiuk-Hojło M. Immunopathogenesis of ophthalmological paraneoplastic syndromes: recent findings. Adv Clin Exp Med. 2018 Oct;27(10):1431-9.

3 Makiyama Y, Kikuchi T, Otani A, Oishi A, Guo C, Nakagawa S, et al. Clinical and immunological characterization of paraneoplastic retinopathy. Invest Ophthalmol Vis Sci. 2013 Aug;54(8):5424-31.

4 Rahimy E, Sarraf D. Paraneoplastic and nonparaneoplastic retinopathy and optic neuropathy: evaluation and management. Surv Ophthalmol. 2013 Sep-Oct;58(5):430-58.
5 Cheng JL, Beebe JD, Nepple KG, Zakharia Y, Mullins RF, Flamme-Wiese MJ, et al. Autoimmune retinopathy and optic neuropathy associated with enolase-positive renal oncocytoma. Am J Ophthalmol Case Rep. 2018 Sep;12:55-60.

6 Adamus G, Champaigne R, Yang S. Occurrence of major anti-retinal autoantibodies associated with paraneoplastic autoimmune retinopathy. Clin Immunol. 2020 Jan;210:108317.

7 Yang S, Dizhoor A, Wilson DJ, Adamus G. GCAP1, Rab6, and HSP27: Novel Autoantibody Targets in Cancer-Associated Retino pathy and Autoimmune Retinopathy. Transl Vis Sci Technol. 2016 May;5(3):1.

8 Golovastova MO, Tsoy LV, Bocharnikova AV, Korolev DO, Gancharova OS, Alekseeva $\mathrm{EA}$, et al. The cancer-retina antigen recoverin as a potential biomarker for renal tumors. Tumour Biol. 2016 Jul;37(7):9899-907.
9 Keltner JL, Thirkill CE, Tyler NK, Roth AM. Management and monitoring of cancer-associated retinopathy. Arch Ophthalmol. 1992 Jan;110(1):48-53.

10 Huynh N, Shildkrot Y, Lobo AM, Sobrin L. Intravitreal triamcinolone for cancer-associated retinopathy refractory to systemic therapy. J Ophthalmic Inflamm Infect. 2012 Sep; 2(3):169-71.

11 Ferreyra HA, Jayasundera T, Khan NW, He S Lu Y, Heckenlively JR. Management of autoimmune retinopathies with immunosuppression. Arch Ophthalmol. 2009 Apr;127(4): 390-7.

12 Espandar L, O'Brien S, Thirkill C, Lubecki LA, Esmaeli B. Successful treatment of cancer-associated retinopathy with alemtuzumab. J Neurooncol. 2007 Jul;83(3):295-302. 